\title{
Effect of sulphasalazine in patients with active Crohn's disease: a controlled double-blind study
}

\author{
P A M VAN HEES*, H J J VAN LIER, PH VAN ELTEREN, W M M DRIESSEN, \\ R A VAN HOGEZAND, G P M TEN VELDE, J H BAKKER, J H M VAN TONGEREN \\ From the Department of Internal Medicine, Division of Gastroenterology, St. Radboud Hospital, \\ University of Nijmegen; Department of Internal Medicine, St. Joseph Hospital, Eindhoven; \\ Department of Pharmacy, St. Radboud Hospital, Nijmegen; Statistical Support Center, \\ University of Nijmegen; The Netherlands
}

SUMMARY The response of active Crohn's disease to sulphasalazine (4-6 g per day) has been studied in a placebo-controlled trial. The study was carried out at two hospitals. From August 1977 to August 1979 all patients with established Crohn's disease were examined for their eligibility for the trial. A nine-item index of inflammatory activity was used as the primary measure of response. The variables in this index were serum albumin, ESR, body weight related to height, abdominal mass, temperature, stool consistency, bowel resection, and extraintestinal symptoms related to Crohn's disease. A favourable response to therapy was defined as a decrease of the activity index with $25 \%$ or more at the end of the trial period, compared with the initial value. Twenty-six patients (13 in each treatment group) have been followed up for six months. The response of active Crohn's disease to sulphasalazine was significantly better than to placebo.

Sulphasalazine (SASP) is widely used in the medical treatment of active Crohn's disease but so far only two controlled trials have been published, the results of which are not unequivocal. ${ }^{2}$ We therefore undertook a controlled double-blind study to evaluate the efficacy of SASP in the treatment of patients with active Crohn's disease. When the trial was planned there was no evidence that any medical treatment was effective in Crohn's disease, so the effect of SASP was compared with a placebo.

\section{Methods}

CASE SELECTION AND DIAGNOSTIC CRITERIA FOR CROHN'S DISEASE

The study was carried out at two hospitals. From August 1977 to August 1979 all patients with established Crohn's disease who were seen at or referred to these hospitals and the patients who were

*Address for correspondence: P A M van Hees, MD, Department of Medicine, Division of Gastroenterology, St. Radboud Hospital, Geert Grooteplein Zuid 16, Nijmegen, The Netherlands.

Received for publication 4 December 1980 being followed up and in whom a recurrence or a relapse of Crohn's disease was diagnosed were examined for their eligibility for the trial.

A patient was considered to have Crohn's disease of the small intestine if either of the following criteria were satisfied: chronic inflammatory disease (persisting for more than three months) of the small intestine with either typical radiological appearance ${ }^{3}$ on radiography of the small bowel or with typical histological appearance on full thickness biopsy of the small bowel lesion; ${ }^{4}$ or typical gross appearance of regional enteritis of the small intestine, visualised at operation and confirmed by the finding of a noncaseating granuloma in a mesenteric lymph node.

The diagnosis of Crohn's disease of the colon demanded the presence of at least one of the following criteria; (1) inflammatory disease of the colon with associated small bowel involvement with typical radiological features of Crohn's disease; (2) inflammatory disease of the colon with typical radiological appearance on barium examination ${ }^{3}$ and typical endoscopic ${ }^{5}$ or histological appearance; ${ }^{4}$ (3) segmental inflammatory disease of the colon with sparing of the rectum as judged by a normal rectal 
biopsy (this criterion did not apply to patients using rectally administered prednisolone).

Before entry into the trial the radiographs of each patient were reviewed by a radiologist and two gastroenterologists to confirm the diagnosis of Crohn's disease.

\section{ASSESSMENT OF INFLAMMATORY ACTIVITY}

For the assessment of the inflammatory activity of Crohn's disease and for the evaluation of the effect of therapy an activity index (AI) was used. ${ }^{6}$ The variables in this index are serum albumin. ESR, body weight related to height, abdominal mass, sex, temperature, stool consistency, bowel resection, and extraintestinal lesions related to Crohn's disease. Index values below 110 points are associated with inactive disease, values between 110 and 150 can be regarded as indicating slight activity, between 150 and 210 as moderate and more than 210 as severe inflammatory activity.

\section{CRITERIA FOR EXCLUSION}

The following categories of patients were excluded from the study: patients with proven allergy for sulphasalazine, sulphapyridine, or salicylates; women who were pregnant or who intended to become pregnant during the time of the study; patients with an activity index of less than 140; severely ill patients in whom the risk of placebo treatment was unacceptable in the judgement of an independent panel consisting of a gastroenterologist, a surgeon, and a radiologist; patients in need of surgery because $o$ complications of Crohn's disease such as intestinal obstruction, intra-abdominal fistula or abscess; patients in whom administration of antibiotics or corticosteroids was necessary for other reasons than Crohn's disease; patients with a blind loop, an ileostomy, a proximal colostomy, or an ileorectal anastomosis; patients with liver disease (serum bilirubin or SGPT at least three times the upper normal limit) or renal disease (creatinine clearance $<30 \mathrm{ml} / \mathrm{min}$ ); patients with accompanying disease that might influence the activity index.

STUDY DESIGN AND RANDOMISATION Patients with active Crohn's disease (activity index $\geqslant 140$ ) and who were not excluded by any of the above criteria were assigned to receive either sulphasalazine (plain taklets, $6 \mathrm{~g}$ per day in four divided doses) or placebo after written informed consent had been obtained. To achieve comparable treatment groups a balanced randomisation according to Taves $^{7}$ was used. The main balancing variables were: location of disease; duration of symptoms; previous medical and surgical therapy, and activity index. In patients complaining of adverse effects the dose of the drug could be reduced to $4 \mathrm{~g}$ per day. Sulphasalazine and placebo tablets were identical in internal and external appearance.

The patients were involved in the trial for a period of 26 weeks. At entry into the study and at the end of the trial period the patients were admitted to the hospital and were evaluated by history, physical examination, routine blood studies, small bowel barium enema studies, barium-enema, and colonoscopy. The patients were seen at the outpatient department two, four, six, eight, 12, 16, and 20 weeks after the start of therapy. At entry into the study and at each follow-up visit all items necessary for the calculation of the activity index were collected. As serum albumin is the principal variable in the activity index, serum albumin levels of all patients were determined at one laboratory. This was done to avoid problems related to different reference values of serum albumin.

During the trial period the patients received no other specific treatment. Analgesics, tranquillisers, sedatives, and antacids were permitted. Antidiarrhoeal agents, corticosteroids, antibiotics, and cholestyramine were prohibited.

Compliance with therapy was monitored by determination of the serum levels of SASP and total sulphapyridine (SP), both during hospitalisation and at each follow-up visit.

In both hospitals the patients were usually seen by the same local gastroenterologist. Throughout the study patients and gastroenterologist were unaware of which treatment was being given. The study drugs were packaged, labelled, and dispensed by the pharmacist of the St. Radboud Hospital. The gastroenterologists did not have access to any laboratory data which could reveal the nature of the drug the patient was taking, such as serum levels of SASP, SP, haptoglobin, LDH, and reticulocyte count.

\section{ASSESSMENT OF RESPONSE TO THERAPY}

A favourable response to therapy was defined as a decrease in the activity index of $25 \%$ or more at the end of the trial period compared with the initial value. Particularly in the case of patients with severe Crohn's disease it was necessary to define criteria for early withdrawal from the study. These criteria were: persistence of daily fever of $38.5^{\circ} \mathrm{C}$ or greater for more than two weeks; daily fever between $38 \cdot 0$. and $38.5^{\circ} \mathrm{C}$ for more than four weeks without a tendency to fall; rise in the activity index of $25 \%$ or more to above 200 points continuing for over two weeks; or an activity index of greater than 200 points for more than eight weeks. Early withdrawal because of one of these reasons was regarded as treatment failure. 
STATISTICAL DESIGN AND ANALYSIS

The trial was designed as a two-stage procedure. It was planned to perform an interim analysis of the first 30 treatment results: 15 from placebo and 15 from SASP-treated patients. The trial was to be stopped if these results were significantly in favour of SASP according to Fisher's exact test (one-sided) at the $1 \%$ level of significance. If not, the trial should be continued with another 30 patients (15 placebo, 15 SASP-treated). The combined results of all 60 patients should be tested with Fisher's exact test at the $5 \%$ level of significance. By computer simulation with 10000 random experiments it was established that the overall significance level of this procedure was not more than about $3 \%$ and that the probability of detecting a $30 \%$ difference in success probabilities (in favour of SASP) ranged from 67 to $80 \%$ (depending on the individual success probabilities), while the probability of detecting a $40 \%$ difference was at least $90 \%$.

It was planned that 30 patients should be included into the trial within a period of two years. However, the number of patients eligible for the trial was lower than expected. Therefore the interim analysis was carried out in August 1979 when the results of 26 patients were available. On the basis of the results of this analysis (presented in this paper) it was decided not to continue the trial.

\section{COMPOSITION OF TREATMENT GROUPS}

From August 1977 to August 197968 patients were examined for their eligibility for the trial. Forty-one patients were excluded because of the following reasons: an activity index of less than 140 points (18 patients); in need of surgery (18 patients); ileostomy

\section{Table 1 Comparability of treatment groups}

\begin{tabular}{lll}
\hline Variable & $\begin{array}{l}\text { Sulphasalazine } \\
(n=13)\end{array}$ & $\begin{array}{l}\text { Placebo } \\
(n=13)\end{array}$ \\
\hline Male/female & $5 / 8$ & $5 / 8$ \\
Age (yr) (mean \pm SD) & $34 \cdot 3 \pm 17 \cdot 0$ & $32 \cdot 9 \pm 14 \cdot 8$ \\
Duration of symptoms $(\mathrm{yr})$ & 5 & 5 \\
$<1$ & 2 & 4 \\
$\mathbf{1 - 3}$ & 6 & 4 \\
$>3$ & 7 & 5 \\
Location of Crohn's disease & 1 & 3 \\
$\quad$ Small bowel only & 5 & 5 \\
$\quad$ Small bowel and colon & 1 & 2 \\
$\quad$ Colon only & 0 & 1 \\
Previous resection for Crohn's disease & 1 & 1 \\
Treated with corticosteroids & 3 & $165 \pm 22$ \\
Treated with sulphasalazine & $185 \pm 30$ & \\
AI at randomisation (mean \pm SD) & 185 \\
\hline
\end{tabular}

or colostomy (two patients); not co-operative (one patient); severely ill (one patient), and other disease influencing the activity index (one patient). Twentyseven patients fulfilled all intake criteria and were randomised to the study. One patient dropped out early because of drug toxicity. Thus 26 patients remained, 13 in each treatment group. Table 1 demonstrates that the patients in the two treatment groups were closely similar in respect of sex distribution, age, duration of symptoms, anatomical site of Crohn's disease, previous resection for Crohn's disease, and activity index at the time of randomisation. One patient received prednisone and four patients SASP. These drugs were withdrawn at least two weeks before entry into the trial.

\section{Results}

The results of the study are summarised in Table 2 . The proportion of patients with a favourable

Fig. 1 Proportional change in activity index $(A I)$ compared with the initial $A I$ week-by-week of the patients in both treatment groups

$\triangle A I(\%)=100 . \frac{A I(t)-A I(o)}{A I(0)}$

$A I(o)=A I$ at entry; $A I(t)=A I t$ weeks after entry into the trial. Median, first, and third quartiles of a sample are the values below which there are 50,25 , and $75 \%$ of the observations, respectively. Median and quartiles of $\triangle A I$ are given instead of mean $\pm S D$ because the latter could not be computed for values of $t$ where $A I$ values of patients who were withdrawn early from the trial are missing. Median and quartiles can still be computed for those $t$ assuming that the missing values

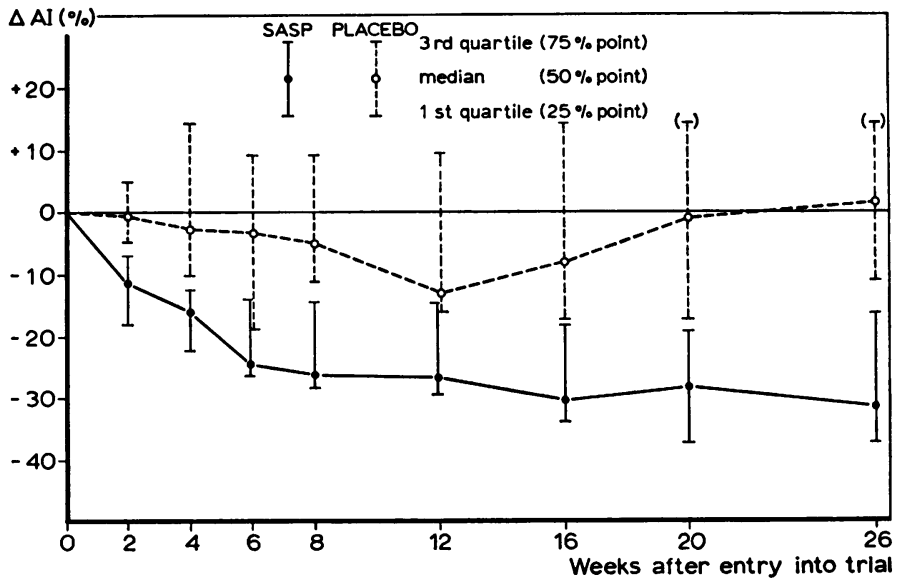
belong to the upper or lower $25 \%$-tail of the distribution. That applies to this study as the patients withdrawn early had the highest positive or the lowest negative $\triangle A I$ values. For the placebo group at 20 and 26 weeks too many values were missing to obtain the third quartile. 
Table 2 Results of study in 26 patients

\begin{tabular}{|c|c|c|c|c|}
\hline \multirow{2}{*}{$\begin{array}{l}\text { Type of } \\
\text { therapy }\end{array}$} & \multirow{2}{*}{$\begin{array}{l}\text { No. of } \\
\text { patients }\end{array}$} & \multicolumn{2}{|c|}{ Favourable response } & \multirow{2}{*}{$\begin{array}{l}\text { Significance } \\
\text { of difference }\end{array}$} \\
\hline & & (no.) & $(\%)$ & \\
\hline $\begin{array}{l}\text { Sulphasalazine } \\
\text { Placebo }\end{array}$ & $\begin{array}{l}13 \\
13\end{array}$ & $\begin{array}{l}8 \\
1\end{array}$ & $\begin{array}{r}61 \cdot 5 \\
7 \cdot 7\end{array}$ & $P=0.0056$ \\
\hline
\end{tabular}

- A favourable response is defined as a fall in the activity index of $25 \%$ or more.

response (a fall in the activity index of $25 \%$ or more) in the SASP group was significantly higher than in the placebo group, $61.5 \%$ versus $7.7 \%$.

The proportional change in the activity index compared with the initial index week-by-week of the patients in both treatment groups is shown in Fig. 1. At the end of the trial period there was a decrease in the activity index of $26 \cdot 2 \pm 15.4 \%$ (mean \pm SD) in the SASP group with an increase of $0.3 \pm 21.2 \%$ in the placebo group. Figure 2 shows the activity index at entry and at the end of the trial period for the individual patients (including those who had to be withdrawn early). In the eight patients in the SASP group with a favourable response the mean fall of the activity index was $36 \cdot 3 \pm 4.7 \%$ (index before and after therapy: $175 \pm 27$ and $111 \pm 13$, respectively). This fall was reached four to eight weeks after the start of the therapy and could be maintained during the remainder of the 26 weeks' follow-up period (Fig. 1). A ninth patient in the SASP group just failed to reach the $25 \%$ limit $(24.2 \%)$. In eight patients treated with SASP the dose of the drug had to be reduced to $4 \mathrm{~g}$ per day because of side-effects; five of them showed a favourable response to therapy. SASP was effective in three of five patients taking $6 \mathrm{~g}$ per day. In the SASP group early withdrawal was necessary in only one patient (because of persistence of the activity index above 200 points for more than eight weeks). This particular patient had severe diarrhoea (five to nine loose stools per day) due to an active Crohn's colitis (activity index: 238 points at entry into the trial). From serum SASP levels and 24 hour urinary excretion of SASP it could be concluded that this patient took the prescribed dose of $6 \mathrm{~g}$ per day. However, serum SP levels were very low: $3 \cdot 5-8 \mu \mathrm{g} / \mathrm{ml}$, as was 24 hour urinary excretion of the split products of SASP; SP and 5amino-salicylic acid (5-ASA): $1 \cdot 2-3 \cdot 2 \%$ and $0 \cdot 3$ $0.6 \%$ of the ingested dose, respectively. This indicates that the larger part of SASP was excreted in the stools because of rapid transit through the colon as a result of diarrhoea. ${ }^{8}$ In the placebo group four patients had to be withdrawn early because of persistence of the activity index above 200 points for more than eight weeks or because of a rise in the index of $25 \%$ or more to above 200 points over two weeks (after four, six, six, and 16 weeks, respectively).

In the SASP group three patients took SASP before the study. Two of them showed a favourable response: a fall of the activity index from 140 to 96 and from 214 to 120 points, respectively. Both patients took $6 \mathrm{~g}$ SASP per day during the trial period, while the dose of the drug before entry into the trial was 2-3 g per day. The third patient took $6 \mathrm{~g}$ SASP before entry into the trial and failed to respond to the same dose during the study.

From the serum levels of SASP and SP it could be concluded that in the SASP group all patients except one took the prescribed dose of the drug. In 10 patients the serum SP levels were above $20 \mu \mathrm{g} / \mathrm{ml}$ throughout the whole trial period and for each patient the serum SP levels during hospitalisation and at outpatient follow-up were about the same. In two patients with severe diarrhoea the serum SP levels were less than $20 \mu \mathrm{g} / \mathrm{ml}$ but the serum SASP levels indicated that they took the prescribed dose. One patient stopped taking SASP six weeks after the start of the therapy after a considerable improvement was reached: a fall in the activity index from 160 to 119 points. The index remained low (118-100 points) for the rest of the trial period.

In this study the disease location within the bowel had no significant influence on the outcome of drug therapy. SASP was effective in five of seven patients with involvement only of the small bowel, in two of five patients with disease confined to the colon, and

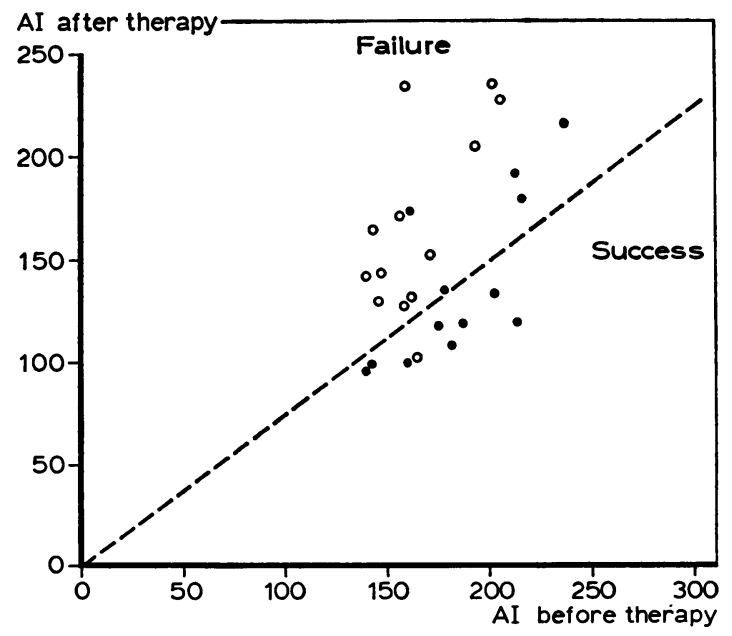

Fig. 2 Activity index (AI) at the end of the trial period (after therapy) plotted against the AI at entry into the study (before therapy) for both treatment groups. Sulphasalazine. $\bigcirc$ Placebo.

- - - Boundary between success (at least $25 \%$ decrease of the AI) and failure of treatment. 
in the only patient with both small and large bowel disease.

It was possible in all patients to maintain the double-blind situation throughout the whole trial period.

\section{Discussion}

SASP has been used for several years in the treatment of patients with active Crohn's disease. However, in contrast with ulcerative colitis, there is little objective evidence of its efficacy in Crohn's disease. At present, only two controlled studies have been published.

A Scandinavian multicentre trial examined SASP versus placebo in two different groups of patients with active Crohn's disease by a double-blind, double-crossover technique with four treatment periods of one month each. ${ }^{1}$ Each patient was treated alternately with SASP ( 3 g per day) and a placebo for one month. The first group consisted of 12 patients with relapse after surgical resection. SASP therapy was not superior to placebo. In a second group of 13 patients without previous surgery only a symptomatic improvement was detected during SASP therapy. The main objection to this study is the short treatment period. Therefore a true difference in favour of SASP could have been missed.

Recently the final results of the American National Co-operative Crohn's Disease Study (NCCDS) have been published. ${ }^{2}$ In a placebo-controlled multicentre trial the response of active Crohn's disease to prednisone $(0.75-0.25 \mathrm{mg} / \mathrm{kg}$ body weight), SASP $(1 \mathrm{~g} / 15 \mathrm{~kg})$, or azathioprine $(2.5 \mathrm{mg} / \mathrm{kg})$ for 17 weeks was studied in 295 patients. Both prednisone and SASP were superior to placebo. SASP proved significantly superior to placebo for patients with colon involvement, whether or not the small bowel was involved, but was not effective against disease confined to the small bowel. The principal criterion of response in the NCCDS study was the Crohn's disease activity index (CDAI). This index is largely determined by subjective variables (abdominal pain, well-being) or variables not necessarily related to inflammatory activity (such as diarrhoea and the use of Lomotil or opiates in patients with ileal resection), while objective parameters of inflammatory activity such as serum albumin and ESR are lacking. ${ }^{9}$ Therefore, the main conclusion that can be drawn from the NCCDS study is that SASP and prednisone are superior to placebo with respect to symptomatic improvement. In Crohn's disease, with its protean manifestations, symptomatic improvement may reflect amelioration of inflammatory activity but this is not necessarily so. The heavy reliance on the CDAI and the method of statistical analysis (a rather complicated ranking scheme of which the clinical relevance is difficult to assess) preclude a definite conclusion about the effect of these drugs on the inflammatory activity on Crohn's disease.

The principal criterion of response in our study was an activity index mainly based on objective criteria reflecting the inflammatory activity of Crohn's disease. Although the number of patients was small, the results demonstrate that SASP (4-6 g per day) was significantly superior to placebo.

We could not confirm the lack of improvement with SASP in patients with only small bowel disease as reported in the NCCDS study. In our patients with small bowel involvement SASP appeared to be at least as effective as in patients with large bowel disease. However, the small number of patients does not allow a definite conclusion.

The diazo bond of SASP is split by colonic bacteria, releasing SP and 5-ASA. ${ }^{10}$ Studies of Azad Khan et al. ${ }^{11}$ and ourselves ${ }^{12}$ suggest that 5-ASA is the active moiety of SASP. In our opinion SASP can also be effective in patients with Crohn's disease of the small bowel because, in these patients, there is often a marked increase in the number of bacteria at the site of inflammation as a result of stasis due to infiltration or stenosis of the intestinal wall. Thanks to this bacterial overgrowth SASP can adequately be split.

This study has shown that 4-6 g SASP is superior to placebo in patients with active Crohn's disease. The optimal dose of SASP and its value as adjunctive therapy to prednisone remains to be determined by further controlled clinical trials.

The authors wish to thank Willemijn van Beek, Mieke van Rens, and Ineke Verschuren for determining sulphasalazine and its metabolites. Sulphasalazine and placebo tablets were supplied by Pharmacia AB, Uppsala, Sweden.

\section{References}

${ }^{1}$ Anthonisen $\mathrm{P}$, Barany $\mathrm{F}$, Folkenborg $\mathrm{O}$, et al. The clinical effect of salazosulphapyridine (SalazopyrinR) in Crohn's disease. A controlled double-blind study. Scand J Gastroenterol 1974; 9: 549-54.

${ }^{2}$ Summers RW, Switz DM, Sessions JT, et al. National cooperative Crohn's disease study: results of drug treatment Gastroenterology 1979; 78: 847-69.

${ }^{3}$ Marshak RH, Lindner AE. Röntgen features of Crohn's disease. Clin Gastroenterol 1972; 1: 411-32.

${ }^{4}$ Morson BC. Pathology of Crohn's disease. Clin Gastroenterol 1972; 1: 265-77.

${ }^{5}$ Geboes K, Vantrappen G. The value of colonoscopy in the diagnosis of Crohn's disease. Gastrointest Edosc 1975; 22: 18-23.

${ }^{6}$ van Hees PAM, van Elteren $\mathrm{Ph}$, van Lier $\mathrm{HJ}$, van Tongeren JHM. An index of inflammatory activity in 
patients with Crohn's disease. Gut 1980; 21 : 279-86. 'Taves DR. Minimization: a new method of assigning patients to treatment and control groups. Clin Pharmacol Ther 1974; 15: 443-53.

${ }^{8}$ van Hees PAM, Tuinte JHM, van Rossum JM, van Tongeren JHM. Influence of intestinal transit time on azo-reduction of salicylazosulphapyridine (Salazopyrin). Gut 1979; 20: 300-4.

${ }^{9}$ Best WR, Bectel JM, Singleton JW, Kern FJr. Development of a Crohn's disease activity index. Gastroenterology 1976; 70: 439-44.
${ }^{10}$ Schöder H, Campbell DES. Absorption, metabolism and excretion of salicylazosulfapyridine in man. Clin Pharm Ther 1972; 13: 539-51.

${ }^{11}$ Azad Khan AK, Piris J, Truelove SC. An experiment to determine the active therapeutic moiety of sulphasalazine. Lancet 1977; 2: 892-5.

${ }^{12}$ van Hees PAM, Bakker JH, van Tongeren JHM. Effect of sulphapyridine, 5-aminosalicylic acid and placebo in patients with idiopathic proctitis. A study to determine the active therapeutic moiety of sulphasalazine. Gut $1980 ; 21$ : 632-5. 